\title{
A multiparametric MRI score for prostate cancer detection: Performance in patients with and without endorectal
} coil

Purpose: To assess the diagnostic performance of multiparametric MRI (mpMRI), in the detection of prostate cancer in two different coil setting: endorectal coil (ERC) versus phased array coil alone (PAC).

Materials and Methods: The study included 302 out of 395 consecutive patients with PSA values between $2.5-4 \mathrm{ng} / \mathrm{ml}$ and an abnormal Digital Rectal Examination (DRE), or patients with PSA values between 4-10 $\mathrm{ng} / \mathrm{ml}$, independently from DRE. Each patient provided informed consent to undergo at serum free/total PSA ratio ( $f / t$ PSA) assay, morphological MRI (mMRI), DWl, MRS, and Trans Rectal Ultrasonography (TRUS) biopsy. The MRI data sets were scored singularly and then mMRI, DWI and MRS data were combined in a single score (CMRI score). cMRI score was correlated to negative biopsies and Gleason score biopsies. ROC curve and McNemar tests were performed.

Results: CMRI score showed high value of sensitivity and NPV for both coil setting ( $84 \%$ and $93 \%$ respectively using ERC, $87 \%$ and $87 \%$ respectively using PAC). CMRI score using ERC did not show statistical superiority compared with cMRI score using PAC alone ( $p$ value at McNemar test $>0.05$ ). A significant correlation was obtained comparing the cMRI score to the Gleason score for both coil conditions.

Conclusions: Pelvic phased array coil imaging of the prostate produces high quality images and the overall performance in prostate cancer detection is equal than those obtained with endorectal coil imaging.

KEYWORDS: prostate cancer - magnetic resonance imaging - spectroscopy - diffusion weighted imaging • gleason score

\section{Introduction}

Prostate cancer diagnosis still represents a clinical challenge, as currently available diagnostic methods remain suboptimal $[1,2]$. On the other side, there are both over-diagnosis and overtreatment risks [3,4] Magnetic resonance imaging (MRI) provides excellent high-contrast, highresolution images of the prostate. In recent years, functional techniques have been applied in order to improve the performance of MRI in the diagnosis of prostate cancer [5-8]. Magnetic resonance spectroscopy (MRS) measures prostate metabolites concentrations, particularly choline and citrate, hat are respectively increased and reduced in cancer [6]. Diffusion Weighted Image (DWI) provides water diffusion properties based imaging, with reduced water diffusion in highly cellular cancer tissues [5]. Early promising data suggest that multiparametric MRI (mpMRI), including morphologic sequences and functional MR approaches, may be of additional value for the localization of prostate cancer and its local staging [8-12].

Literature studies have found that, compared to the use of a phased array coil (PAC) alone, the use of both an endorectal coil (ERC) and a PAC to acquire T2-weighted fast spin-echo images of the prostate at $1.5 \mathrm{~T}$ [13-16] and 3.0T [15] results in greater visibility of anatomical details and more accurate prostate cancer staging. The use of both a PAC and an ERC (a combination referred to hereafter as ERC+PAC) improves prostate imaging by providing greater signalto-noise ratio (SNR) and thus increased spatial resolution. However, the insertion of the ERC causes patient discomfort, is expensive, and can lead to complications such as proctitis [15]. Recently, the availability of higher field strength magnets, increased numbers of phased array receiver coils or multi-channels phased array (8 channels or more), and improved pulsesequence techniques has generated interest in the possibility of performing prostate MRI using only a PAC.

The aim of this study was to evaluate the diagnostic performance of mpMRI in the detection of prostate cancer, including morphological (mMRI), DWI and MRS obtained with an ERC and a PAC compared to mpMRI obtained with only a PAC.

\section{Material and Methods}

\section{Patient selection}

From 2009 to 2011, 395 consecutive male

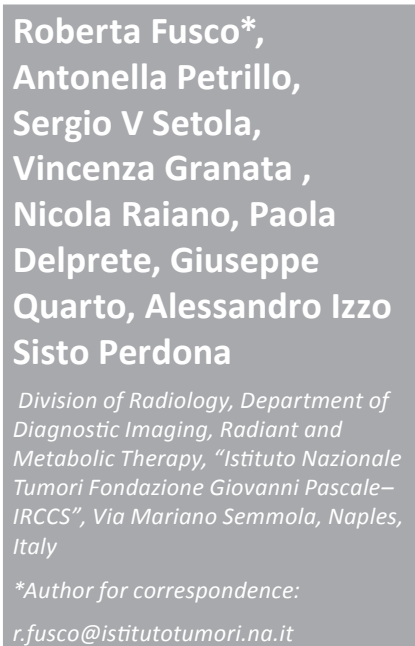


patients were screened to be enrolled in a singlecenter prospective observational study. The inclusion criteria were: PSA values between $2.5-4 \mathrm{ng} / \mathrm{ml}$ and an abnormal DRE, or PSA values between $4-10 \mathrm{ng} / \mathrm{ml}$, independently from DRE. The exclusion criteria were: inability to give informed consent; prior history of prostate cancer excluded, prior pelvic irradiation, previous hormonal or surgical therapy; MRI contraindications (cardiac pacemakers, surgical clips, metallic hip implant); recent rectal surgery, latex allergy.

All eligible patients underwent morphological and functional MRI and TRUS prostate biopsies. A register included every single patient that decided to be enrolled in this approved study. Any patient who decided to undergo the MRI examination signed an explicit informed consent.

\section{- MRI data acquisition}

The MRI was performed before prostate biopsies. The MRI protocol included insertion of an endorectal coil (ERC) (Medrad, Pittsburg, PA, USA), inflated with $60-90 \mathrm{ml}$ of air, and subsequent imaging acquisition using both a 1.5T MRI system (Siemens Symphony Tim, Erlangen, Germany) coupled to a phased-array surface coil or a 8 channel phased-array surface coil (PAC) alone. The MRI total acquisition time was approximately $40 \mathrm{mins}$. The mpMRI included mMRI, MRS and DWI. The mMRI included Turbo Spin Echo (TSE) T2-weighted sequences in three perpendicular planes and coronal and transverse TSE T1-weighted sequence. Transverse TSE T2-weighted sequence parameters were: TR/TE, 3800/104ms (sagittal: 4660/96ms; coronal: 5000/98ms); slice thickness, $3 \mathrm{~mm} /$ gap $0 \mathrm{~mm}$; flip angle, $180^{\circ}$; acquisition matrix, $320 \times 288$ (sagittal and coronal $320 \times 256$ ); field of view (FOV), $240 \times$ $240 \mathrm{~mm}^{2}$. Transverse T2-weighted images were acquired with and without fat saturation.

TSE T1-weighted were acquired in coronal to visualize lymph nodes, with the following sequence parameters: TR/TE, 550/12 ms; slice thickness, $3 \mathrm{~mm} /$ gap $0 \mathrm{~mm}$; flip angle, $150^{\circ}$; acquisition matrix, $256 \times 202$; FOV, $448 \times 512$ $\mathrm{mm}^{2}$. Transverse TSE T1-weighted sequence parameters were: TR/TE, $706 / 7.8 \mathrm{~ms}$, slice thickness, 3mm/gap 0mm; flip angle, $150^{\circ}$; acquisition matrix, $356 \times 192$; field of view (FOV), $240 \times 240 \mathrm{~mm}^{2}$. The MRS parameters were: TR/TE, 690/120 ms; flip angle, $90^{\circ}$. The volume of Interest (VOI) was composed by 16 $\times 16 \times 16$ voxel of $6.25 \times 6.25 \times 6.25 \mathrm{~mm}^{3}$. The transverse echo-planar DWI pulse sequence parameters were: TR/TE, $2700 / 83 \mathrm{~ms}$; slice thickness, $3.56 \mathrm{~mm}$; flip angle, $90^{\circ}$, acquisition matrix; 160x102 and FOV, 136x160 $\mathrm{mm}^{2}$; b value $=0,50,100,150,300,600,800 \mathrm{~s} / \mathrm{mm}^{2}$. Antispasmodic drug was not used.

\section{- Image analysis}

All mpMRI findings were graded by two radiologists with over 10-years of experience in prostate MRI and blinded to both clinical and biochemical data of the patients. A consensus evaluation method for scoring MRI findings was adopted in order to reach more reliability in image interpretation. The scoring system was evaluated on a per-patient basis being not achievable a reasonably accurate evaluation on a per-site basis. A $5 \mathrm{~mm}$ diameter was considered as the inferior threshold for including lesions. The mMRI was scored using a 0-3 scale for peripheral gland $(0=$ No abnormality; $1=$ Geometric hypointense area, with low cancer suspicion; $2=$ Diffuse non nodular hypointense area, with intermediate cancer suspicion; $3=$ nodular hypointense area, with high cancer suspicion) and by using a $0-2$ scale for the central gland $\quad(0=$ Heterogeneous well-marginated nodules; $1=$ Homogeneously hypointense well-marginated nodules; $2=$ Homogeneously hypointense ill-marginated nodules) [16-21].

The MRS raw data were elaborated using Syngo MR-B17 spectroscopy package (Siemens, Erlangen, Germany). The software generated spectra and localized corresponding voxels on superimposed T2-weighted images. The MRS was rated as diagnostic or non-diagnostic if the metabolites spectrum was good, after resolving metabolic resonances, and limited baseline distortions, due to residual water or lipids, were present. For each voxel the software calculated areas under citrate, choline and creatine peaks and Choline+Creatine/Citrate peak ratio. Diagnostic spectra were considered negative or positive, and scored as 0 or 1 (sMRI score) respectively, in relation to Choline+Creatine/ Citrate ratio threshold of 0.86 , as reported in the literature [6].

The DWI data and Apparent Diffusion Coefficient (ADC) maps were elaborated using Syngo MR-B17 diffusion package (Siemens, 
Erlangen, Germany). DWI was considered positive when focal areas, characterized by persistent signal intensity at b-value increase and/or ADC map hypointensity in relation to the adjacent gland, were evident [19]. DWI was scored respectively as 0 or 1 (dMRI score) if a negative or positive finding was reported.

A multiparametric combined score (cMRI score) was obtained for every site as the sum of scores from every MRI technique. For every derived score, the highest site score was used as patient reference score. The cut-off for cMRI was 2 as obtained in a previous studies [10].

\section{- Biopsy and pathological analysis}

TRUS biopsy was performed as previously described in literature [11], by an expert urologist with a 10-years experience. A 12-site biopsy scheme ( 2 cores, paramedian and lateral, from base, midgland and apex, bilaterally) was adopted to cover the peripheral prostate, using a biopsy gun armed with a 18-Gauge needle. In patients with a prostate volume $>45 \mathrm{cc}$ four additional cores were acquired from the central gland (superiorly and inferiorly, bilaterally). The urologist was blinded to the MRI findings during the prostatic biopsy.

A pathologist with more than 10 years' experience in genito-urinary pathology evaluated biopsy cores. Gleason scores were considered significant if at least cancer grade $\geq$ 4 was evident. In case of a negative biopsy, the patient was scheduled for a follow-up visit in a year. When high-grade prostatic intraepithelial neoplasia (HG-PIN) or atypical small acinar proliferation (ASAP) were diagnosed, the biopsy was repeated after 3-6 months using the 16-sites (12 peripheral and 4 central sites) scheme. If the repeat biopsy was negative for cancer, the patient was scheduled for a follow-up visit at 1 year.

Patients were considered positive at biopsy if cancer was reported in at least one core, independently from the Gleason grade. The highest Gleason grade reported at biopsy was considered as patients' reference Gleason grade value.

\section{- Statistical Analysis}

Fishers exact test was used to evaluate statistical significance of Decision matrix (DM) tables. Sensitivity, specificity, positive and negative predictive value (PPV and NPV) were calculated for cMRI score, using DM. Matched sample tables and McNemar test were used to compare diagnostic performance for different coil setting (ERC versus PAC). Spearman's Rank Correlation Coefficient was performed on a per-patient basis correlating patients' reference scores derived from MRI data sets with the results of biopsies (Gleason score). A p value $<0.05$ was considered significant. All analysis were performed using Statistic Toolbox of Matlab R2007a. Statistical correlation (TABLE 4).

\section{Results}

Overall, 302 patients were enrolled in the study, 136 acquired with endorectal coil (FIGURES 1 and 2) and 166 acquired with multi-channel phased array coil (FIGURES 3 and 4). TABLE 1 reported patient's characteristics for both coil setting. Fishers exact tests showed statistical significance for each DM table with a $\mathrm{p}$ value always $<0.05$. TABLE 2 reports diagnostic performance of cMRI score using different coil setting. cMRI score showed high value of sensitivity and NPV for both coil setting (84\% and $93 \%$ respectively using ERC, $87 \%$ and $87 \%$ respectively using PAC). cMRI score using ERC did not show statistical superiority compared with cMRI score using PAC alone ( $\mathrm{p}$ value at McNemar test >0.05). TABLE 3 showed Spearman's Rank Correlation

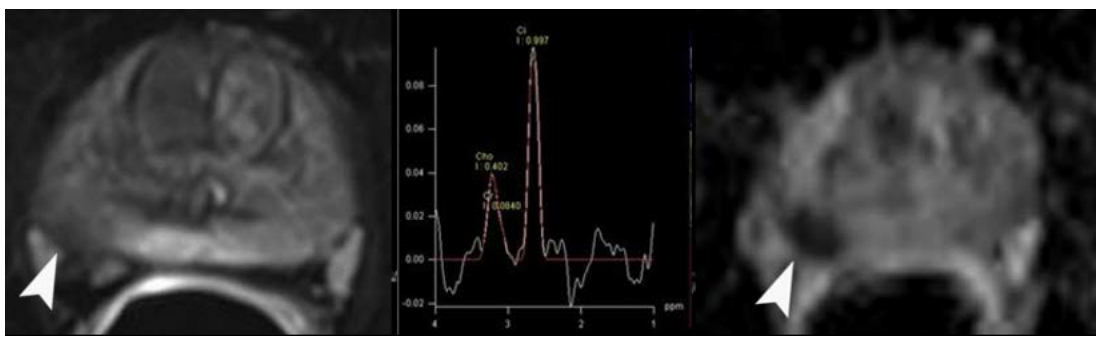

Figure 1. Transverse TSE T2 weighted sequence (left image; TR/TE, 3800/104 ms; flip angle, $180^{\circ}$ ) showed a small ill defined nodular hypointense area $(\mathrm{mMRI}=3)$ in the right peripheral midgland (arrowhead). MRS was negative, while positive findings were evident on ADC maps (right image, arrowhead). cMRI score was 4 . Gleason score was 8. 


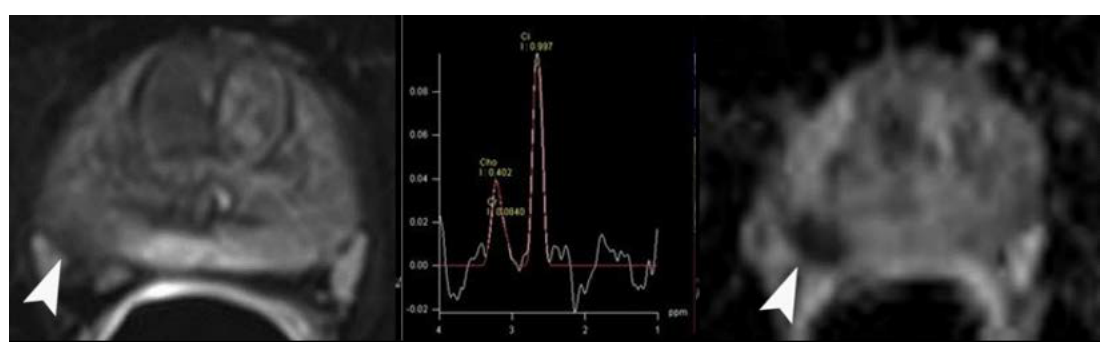

Figure 2. On transverse T2 weighted sequence (left image; TR/TE, 3800/104 ms; flip angle, $180^{\circ}$ ) a diffuse hypointense area $(m M R I=2)$, particularly in the right peripheral midgland (arrowhead) was evident. MRS was positive, and corresponding ADC map showed hypointense areas (right image, arrowhead). cMRI score was 4 . Gleason score was 8.

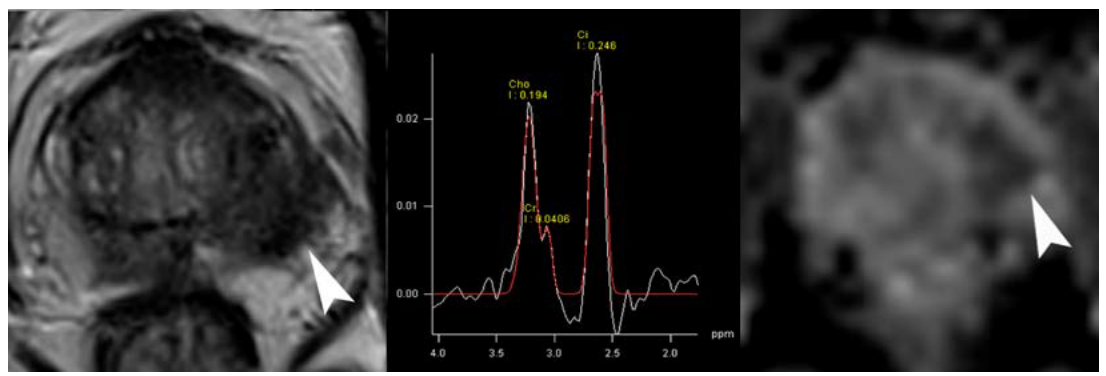

Figure 3. Transverse $T 2$ weighted sequence (left image) shows an ill defined nodular hypointense area $(\mathbf{m M R I}=3)$ in the left peripheral midgland (arrowhead). Positive findings were evident on MRS and on ADC maps (right image, arrowhead). cMRI score was 5. Gleason score was 7.

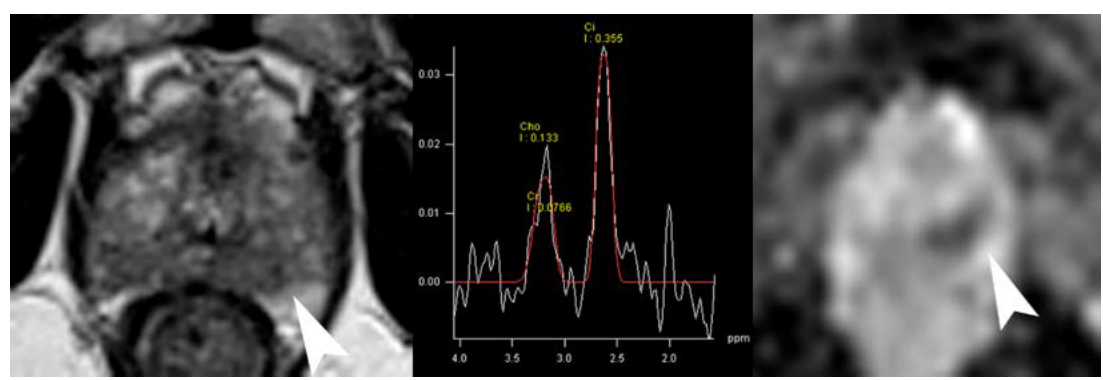

Figure 4. On transverse $T 2$ weighted sequence (left image) a diffuse hypointense area $(m M R I=2)$ in the left peripheral apex (arrowhead) is evident. MRS was negative while ADC map showed a hypointense area (right image, arrowhead). cMRI score was 3. Gleason score was 7.
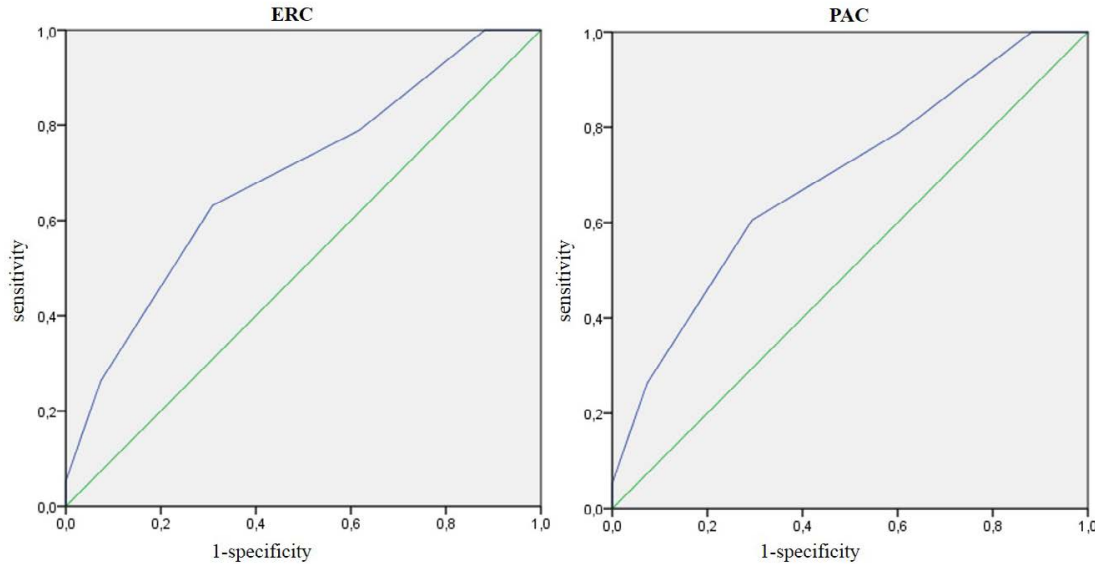

Figure 5. ROC curves for CMRI score in two coil setting.

Coefficients for each score using ERC and PAC. A significant correlation was obtained comparing the cMRI score to the Gleason score for both coil conditions (FIGURE 5). 
Table 1. Patients characteristics.

\begin{tabular}{|ccc|}
\hline Variables & ERC & PAC \\
\hline Patients, $n$ & 136 & 166 \\
\hline Age, years & $66.35(8.4)$ & $64.23(8.6)$ \\
\hline PSA, $n g / m l$ & $6.8(2.4)$ & $6.6(2.3)$ \\
\hline f/t PSA, \% & $18.5(7.3)$ & $19.1(6.9)$ \\
\hline Values are expressed as a mean (SD) & & \\
\hline
\end{tabular}

\section{Table 2. Diagnostic performance.}

\begin{tabular}{cccccc} 
Variables & Sensitivity (\%) & Specificity (\%) & PPV (\%) & NPV (\%) & AUC \\
\hline CMRI with ERC & 84 & 50 & 28 & 93 & 0.74 \\
\hline CMRI with PAC & 87 & 50 & 49 & 87 & 0.72
\end{tabular}

Abbreviations: cMRI: combined MRI score; PPV: positive predictive value; NPV: negative predictive value; AUC: area under ROC curve

\begin{tabular}{|c|c|c|c|c|c|c|}
\hline \multicolumn{2}{|l|}{ Variables } & \multirow{2}{*}{$\begin{array}{c}\text { mMRI Score } \\
1.000\end{array}$} & \multirow{2}{*}{$\begin{array}{c}\text { dMRI Score } \\
.304^{* *}\end{array}$} & \multirow{2}{*}{$\begin{array}{c}\text { sMRI Score } \\
.053\end{array}$} & \multirow{2}{*}{$\begin{array}{c}\text { cMRI Score } \\
.901^{* *}\end{array}$} & \multirow{2}{*}{$\begin{array}{c}\text { Gleason Score } \\
.424^{* *}\end{array}$} \\
\hline \multirow{10}{*}{$\begin{array}{l}\text { Rho } \\
\text { Spearman's } \\
\text { Coefficient }\end{array}$} & \multirow{2}{*}{ mMRI Score } & & & & & \\
\hline & & - & .000 & .502 & .000 & .000 \\
\hline & \multirow{2}{*}{ dMRI Score } & $.304^{* *}$ & 1.000 & $-.262^{* *}$ & $.509^{* *}$ & -.011 \\
\hline & & .000 & - & .001 & .000 & .886 \\
\hline & \multirow{2}{*}{ sMRI Score } & .053 & $-.262^{* *}$ & 1.000 & $.311^{* *}$ & $.345^{* *}$ \\
\hline & & .502 & .001 & - & .000 & .000 \\
\hline & \multirow{2}{*}{ cMRI Score } & $.901^{* *}$ & $.509^{* *}$ & $.311^{* *}$ & 1.000 & $.668^{* *}$ \\
\hline & & .000 & .000 & .000 & - & .000 \\
\hline & \multirow{2}{*}{$\begin{array}{l}\text { Gleason } \\
\text { Score }\end{array}$} & $.424^{* *}$ & -.011 & $.345^{* *}$ & $.6608^{*}$ & 1.000 \\
\hline & & .000 & .886 & .000 & .000 & - \\
\hline \multicolumn{7}{|c|}{$* * p$ value $<0.01$} \\
\hline \multicolumn{7}{|c|}{ * p value $<0.05$} \\
\hline
\end{tabular}

\begin{tabular}{|c|c|c|c|c|c|c|}
\hline \multicolumn{2}{|l|}{ Variables } & \multirow{2}{*}{$\begin{array}{c}\text { mMRI Score } \\
1.000\end{array}$} & \multirow{2}{*}{$\begin{array}{c}\text { dMRI Score } \\
.195^{*}\end{array}$} & \multirow{2}{*}{$\begin{array}{c}\text { sMRI Score } \\
.035\end{array}$} & \multirow{2}{*}{$\begin{array}{c}\text { CMRI Score } \\
.847^{* *}\end{array}$} & \multirow{2}{*}{$\begin{array}{c}\text { Gleason Score } \\
.206^{*}\end{array}$} \\
\hline \multirow{10}{*}{$\begin{array}{l}\text { Rho } \\
\text { Spearman's } \\
\text { Coefficient }\end{array}$} & \multirow{2}{*}{ mMRI Score } & & & & & \\
\hline & & - & .045 & .719 & .000 & .034 \\
\hline & \multirow{2}{*}{ dMRI Score } & $.195^{*}$ & 1.000 & -.071 & $.508^{* *}$ & $.283^{* *}$ \\
\hline & & .045 & & .471 & .000 & .003 \\
\hline & \multirow{2}{*}{ sMRI Score } & .035 & -.071 & 1.000 & $.389^{* *}$ & $.283^{* *}$ \\
\hline & & .719 & .471 & & .000 & .003 \\
\hline & \multirow{2}{*}{ cMRI Score } & $.847^{* *}$ & $.508^{* *}$ & $.389^{* *}$ & 1.000 & $.650^{* *}$ \\
\hline & & .000 & .000 & .000 & - & .000 \\
\hline & \multirow{2}{*}{$\begin{array}{l}\text { Gleason } \\
\text { Score }\end{array}$} & $.206^{*}$ & $.283^{* *}$ & $.283^{* *}$ & $.650^{* *}$ & 1.000 \\
\hline & & .034 & .003 & .003 & .000 & \\
\hline \multicolumn{7}{|c|}{ ** p-value $<0.01$} \\
\hline \multicolumn{2}{|c|}{${ }^{*} \mathrm{p}$-value $<0.05$} & & & & & \\
\hline
\end{tabular}

\section{Discussion}

Many studies investigated MRI performances for the diagnosis of prostate cancer. Because prostate MRI interpretation can be subjective and inconsistent and mMRI alone showed a wide variability of sensitivity and specificity [4,21], different techniques, such as MRS and DWI, were proposed [4-14]. Combining mMRI with functional approaches improves MRI specificity [22-25], particularly when using an integrated data analysis with scoring system [24-25]. Prostate Imaging and Reporting Archiving Data System [PI-RADS] have been developed on a 1- to 5-point scale for improved standardization 
of MRI interpretation and reporting [24] using a multi-parametric approach. A recent metaanalysis of 14 studies evaluating use of the PI-RADS scoring system for prostate cancer detection on multi-parametric MRI showed good diagnostic accuracy [25].

In our previous study [10] we demonstrated that the cMRI score had higher sensitivity and higher NPV than either single techniques (mMRI, DWI and MRS), or their combinations in couples (mMRI/MRS score and mMRI/DWI score). Moreover, it was superior to $\mathrm{f} / \mathrm{t}$ PSA. The cMRI score was also better in differentiating negative biopsies and significant or insignificant Gleason score positive biopsies. A significant correlation was observed between cMRI score and Gleason score, with all significant Gleason score tumors showing a cMRI score $\geq 2$. Objective of this study was to evaluate the diagnostic performance of mpMRI in the detection of prostate cancer, including mMRI, DWI and MRS obtained with an ERC and a PAC compared to mpMRI obtained with only a PAC.

Generally, use of a higher field strength (3.0 $\mathrm{T}$ instead of $1.5 \mathrm{~T}$ ) ore use of endorectal coil improved the detection sensitivity for extracapsular extension (ECE) and seminal vesicle invasion (SVI) detection [8]. ERC use appeared useful for a field strength of $1.5 \mathrm{~T}$ in the absence of multiparametric MRI. An ERC is inserted tightly against the prostate during MRI examination in order to increase image resolution and improve staging accuracy [2629].

The results of our study show that pelvic phased array coil imaging of the prostate produces high quality images (FIGURES 3 and 4 versus FIGURES 1 and 2 ) and that the overall performance in the detection of prostate cancer is equal than those obtained with endorectal coil imaging (TABLES 2 and 3). These results are similar at those reported by Lee et al. [26] that concluded that in terms of diagnostic accuracy and comfort of patients, the use of ERC did not significantly improve the staging of prostate cancer and presented several complications; therefore, phased-array coil MRI is a better alternative considering comorbidity. Different results were reported from Baur et al. [5]: T2 weighted imaging and DWI had a range of area under the curve with a PAC and with ERC-
PAC of 0.95-0.99 and 0.93-0.97, respectively $(\mathrm{p}=0.1395)$. They concluded that $\mathrm{T} 2$ weighted imaging and DWI performed at 3T for prostate cancer lesion identification and evaluation did not differ significantly with both coil setups and that patients preferred MRI without an ERC. Instead, our results are in contrast compared to the findings of Fütterer et al. [27] and Costa et al. [30]. Futterer et al. [27] showed an accuracy and a specificity significantly better with endorectalpelvic phased-array coils $(\mathrm{P}<0.05)$. The overall staging accuracy, sensitivity and specificity for the retrospective readers were $78-79 \%, 56-58 \%$ and $96 \%$, for the endorectal-pelvic phasedarray coils. Area under the ROC curve (Az) was significantly higher for endorectal-pelvic phased-array coils $(\mathrm{Az}=0.74)$ compared to pelvic phased-array coil $(\mathrm{Az}=0.57)$, for the prospective reader. Costa et al. [30] showed that the use of combined ERC and pelvic phased-array coil for T2-weighted imaging and diffusion-weighted imaging with 3T MR scanner provides superior sensitivity for the detection of prostate cancer (78\%) compared to an examination performed without the ERC (43\%).

However, a well recognized limitation of endorectal coil imaging is the presence of artefacts which degrade image quality, but this drawback has not been emphasized as an important cause of errors in the radiological literature. The changes in prostate shape and volume after the introduction of an endorectal coil may cause difficulties in MRI-computed tomography fusion and radiotherapy planning [31]. In one recent study reported by Jager et al. [32] artefacts were considered to be the prime cause of low quality images in $14 \%$ of cases. Artefacts encountered in ERC imaging include coil flare, which is seen at the interface of the anterior aspect of the balloon and normal tissue, and appears as a band or flare of high signal intensity on all sequences. The coil related "straight line" artefact is seen as a line of high signal intensity across the image in the transaxial plane. Rectal movement also degrades image quality by producing movement artefacts across the image, resulting in poor definition of anatomical structures. Although signal intensity post-processing algorithms which reduce these artefacts are available from some manufacturers (and third party suppliers), these algorithms vary widely in quality and are therefore not uniformly reliable. 
Recent developments in sequence design with the introduction of turbo fast spin echo sequences and in design of pelvic phased array coils have undoubtedly improved image quality of prostate cancer imaging. Phased array coil imaging has the advantage that the anterior pelvis, bladder and pelvic lymph nodes can be evaluated with high resolution images and that the technique is non-invasive. Although the endorectal coil can be combined with a PAC coil as part of a multicoil array [13], use of this facility has been slow to develop in clinical practice and hence experience remains limited.

Another limitation is that patients with rectal stenosis or immediately after surgery or radiotherapy may not be good candidates for the use of the endorectal coil during MR examination. When higher field strengths or phased array coil multichannel (8 channel or more) and additional functional techniques were used, studies that used an ERC showed lower sensitivity and heterogeneous specificity than studies without an ERC [8]. Lee et al. [26] reported that the use of ERC MRI did not significantly improve the staging of prostate cancer (AUC 0.67 versus 0.66 respectively with and without ERC) and presented several complications in $11.4 \%$ of patients. Margolis et al. [17] reported that an endorectal coil is not absolutely necessary and that the utility will depend on the performance of the scanner in question. Rectal distention with the associated susceptibility can markedly degrade DWI and potentially MRSI. An 8-channel external phased array could replace the use of an endorectal coil and the use of additional functional imaging techniques seemed to improve the accuracy of local staging $(17,26)$. Also the ESUR prostate MR guidelines 2012 reported in acquisition protocols minimum requirements that imaging can adequately be performed at $1.5 \mathrm{~T}$ using a good 8 to 16-channel pelvic phased array [33].

Considering our results the use of PAC alone for morphological and functional MRI acquisition did not limit overall images quality and diagnostic accuracy and it should be prefer for patient comfort.

A single limitation of the study is reported.
MRI was compared to TRUS biopsy. Even if TRUS biopsy is reported to have a low diagnostic accuracy, it remains the only current diagnostic technique for the diagnosis of prostate cancer. The purpose of our study was to evaluate the diagnostic performance of the scoring system for patients work-up, where TRUS biopsy is the gold standard. When surgery is used as standard reference in a study, a large selection bias is introduced because many patients can be selected for non-surgical treatment or active surveillance [34], as occurred in our population. According to the literature, we adopted a consensus evaluation method for scoring MRI findings in order to have more reliability in image interpretation [25]. We evaluated the scoring system on a per-patient basis because a reasonably accurate evaluation on a per-site basis was not achievable [34]. As reported [22], during needle biopsy the path does not usually correspond to any MRI plane. Moreover, biopsy tumour localization is affected by cores classification according to the needle entry site, without considering the real needle path [35]. Furthermore, in our study, the urologist was blinded to the MRI findings during the prostatic biopsy. Further investigation should be performed to obtain reslicing and registration of MR images in order to reliably correlate MRI findings to TRUS biopsy.

\section{Conclusion}

In conclusion, the cMRI score showed high accuracy both in term of sensitivity than in terms of NPV independently if an endorectal coil is used or a phased array coil alone. Therefore, the use of an ERC may be omitted in a prostate cancer detection setting and multi-channel phased-array coil MRI is a better alternative considering comorbidity.

\section{Funding}

This study was not funded.

\section{Conflict of Interest}

All Authors declare that have no conflict of interest.

\section{Informed consent}

Informed consent was obtained from all individual participants included in the study. 


\section{REFERENCES}

1. Cooperberg MR, Lubeck DP, Meng MV, et al. The changing face of low-risk prostate cancer: Trends in clinical presentation and primary management. J. Clin. Oncol. 22, 2141-2149 (2004).

2. Thompson IM, Pauler DK, Goodman PJ, et al. Prevalence of prostate cancer among men with a prostate specific antigen level $\leq 4.0 \mathrm{ng}$ per millilitre. N. Engl.J. Med. 350, 2239-2246 (2004).

3. Draisma G, Boer R, Otto SJ, et al. Lead times and overdetection due to prostate-specific antigen screening: Estimates from the European Randomized Study of Screening for Prostate Cancer. J. Natl. Cancer. Inst. 95, 868-878 (2003).

4. Sanders A, Buchan N. Infection-related hospital admissions after transrectal biopsy of the prostate. ANZ. J. Surg. 83, 246-248 (2013)

5. Baur $\mathrm{AD}$, Daqqaq $\mathrm{T}$, Wagner $\mathrm{M}$, et al. T2and diffusion-weighted magnetic resonance imaging at 3T for the detection of prostate cancer with and without endorectal coil: An intraindividualcomparison of image quality and diagnostic performance. Eur. J. Radiol. 85, 1075-1084 (2016).

6. Javali TD, Dwivedi DK, Kumar R, et al. Magnetic resonance spectroscopy imagingdirected transrectal ultrasound biopsy increases prostate cancer detection in men with prostatespecific antigen between $4-10 \mathrm{ng} / \mathrm{mL}$ and normal digital rectal examination. Int. J. Urol. 21, 257-262 (2014).

7. Sankineni S, Osman M, Choyke PL. Functional MRI in Prostate Cancer Detection. Biomed. Res. Int. 14, 1-8, (2014).

8. Loffroy R, Chevallier O, Moulin M, et al. Current role of multiparametric magnetic resonance imaging for prostate cancer. Quant. Imaging. Med. Surg. 5, 754-764 (2015).

9. Hegde JV, Mulkern RV, Panych LP, et al. Multiparametric MRI of Prostate Cancer: An Update on State-of-the-Art Techniques and Their Performance in Detecting and Localizing Prostate Cancer. J. Magn. Reson. Imaging. 37, 1035-1054 (2013).

10. Petrillo A, Fusco R, Setola SV, et al. Multiparametric MRI for prostate cancer detection: performance in patients with prostate-specific antigen values between 2.5 and $10 \mathrm{ng} / \mathrm{mL}$. J. Magn. Reson. Imaging. 39, 12061212 (2014).

11. Perdonà S, Di Lorenzo G, Autorino R, et al. Combined magnetic resonance spectroscopy and dynamic contrast-enhanced imaging for prostate cancer detection. Urol. Oncol. 31, 761765 (2013).

12. Fusco R, Sansone M, Petrillo M, et al.
Multiparametric MRI for prostate cancer detection: Preliminary results on quantitative analysis of dynamic contrast enhanced imaging, diffusion-weighted imaging and spectroscopy imaging. Magn. Reson. Imaging. 34, 839-45 (2016).

13. Hricak $\mathrm{H}$, White $\mathrm{S}$, Vigneron $\mathrm{D}$, et al. Carcinoma of the prostate gland: MR imaging with pelvic phased-array coils versus integrated endorectal-pelvic phased-array coils. Radiology. 193, 703-709 (1994).

14. Futterer JJ, Engelbrecht MR, Jager GJ, et al. Prostate cancer: comparison of local staging accuracy of pelvic phased-array coil alone versus integrated endorectal-pelvic phased-array coils. Local staging accuracy of prostate cancer using endorectal coil MR imaging. Eur. Radiol. 17, 1055-1065 (2007).

15. Heijmink SW, Futterer JJ, Hambrock T, et al. Prostate cancer: body-array versus endorectal coil MR imaging at $3 \mathrm{~T}$-comparison of image quality, localization, and staging performance. Radiology. 244, 184-195 (2007)

16. Turkbey B, Albert PS, Kurdziel K, et al. Imaging localized prostate cancer: current approaches and new developments. Am. J. Roentgenol. 192, 1471-1480 (2009).

17. Margolis DJA. Multiparametric MRI for Localized Prostate Cancer: Lesion Detection and Staging. BioMed. Res. Intl. 14, 1-11 (2014).

18. Shukla-Dave A, Hricak H, Kattan MW, et al. The utility of magnetic resonance imaging and spectroscopy for predicting insignificant prostate cancer: an initial analysis. BJU. 99, 786-793 (2007)

19. Lim HK, Kim JK, Kim KA, et al. Prostate cancer: apparent diffusion coefficient map with T2-weighted images for detection-amultireader study. Radiology. 250, 145-151 (2009).

20. Akin O, Sala E, Moskowitz CS, et al. Transition zone prostate cancers: features, detection, localization, and staging at Endorectal MR Imaging. Radiology. 239, 784-792 (2006).

21. Rooij DM, Hamoen EH, Witjes JA, et al. Accuracy of Magnetic Resonance Imaging for Local Staging of Prostate Cancer: A Diagnostic Meta-analysis. Eur. Urol. 70, 233-245 (2016).

22. Cirillo S, Petracchini M, Della Monica P, et al. Value of endorectal MRI and MRS in patients with elevated prostate-specific antigen levels and previous negative biopsies to localize peripheral zone tumours. Clin. Radiol. 63, 871-879 (2008).

23. Umbehr M, Bachmann LM, Held U. Combined magnetic resonance imaging and magnetic resonance spectroscopy imaging in the diagnosis of prostate cancer: a systematic review and metaanalysis. Eur. Urol. 55, 575-590 (2009).

24. Abd-Alazeez M, Ahmed HU, Arya M, et al. The accuracy of multiparametric MRI in men with negative biopsy and elevated PSA level-can it rule out clinically significant prostate cancer? Urol. Oncol. 32, 17-22 (2014).

25. Hamoen EH, de Rooij M, Witjes JA, et al. Use of the prostate imaging reporting and data system (PI-RADS) for prostate cancer detection with multiparametric magnetic resonance imaging: A diagnostic meta-analysis. Eur. Urol. 67, 1112-1121, (2005).

26. Lee SH, Park KK, Choi KH, et al. Is endorectal coil necessary for the staging of clinically localized prostate cancer? Comparison of nonendorectal versus endorectal MR imaging. World. J. Urol. 28, 667-672 (2010).

27. Fütterer JJ, Engelbrecht MR, Jager GJ, et al. Prostate cancer: comparison of local staging accuracy of pelvic phased-array coil alone versus integrated endorectal-pelvic phased-array coils. Local staging accuracy of prostate cancer using endorectal coil MR imaging. Eur. Radiol. 17, 1055-1065 (2007).

28. Westphalen AC, Coakley FV, Qayyum A, et al. Peripheral zone prostate cancer: accuracy of different interpretative approaches with MR and MR spectroscopic imaging. Radiology. 246, 177-184 (2007)

29. Chen H, Sutedjo J, Wang L, et al. Prostate Cancer Magnetic Resonance Spectroscopy Imaging at 1.5 and 3.0T: A Meta-Analysis. Technol. Cancer. Res. Treat. 15, 625-631 (2016).

30. Costa DN, Yuan Q, Xi Y, Rofsky NM, et al. Comparison of prostate cancer detection at 3-T MRI with and without an endorectal coil: A prospective, paired-patient study. Urol. Oncol. 34, 257-213 (2016).

31. Shah ZK, Elias SN, Abaza R, et al. Performance comparison of $1.5 \mathrm{~T}$ endorectal coil MRI with 3.0T nonendorectal coil MRI in patients with prostate cancer. Acad. Radiol. 22, 467-474 (2015).

32. Jager GJ, Ruijter ET, Van de KCA, et al. Local staging of prostate cancer with endorectal MR imaging: correlation with histopathology. Am. J. Roentgenol. 166, 845-852 (1996).

33. Barentsz JO, Richenberg J, Clements R, et al. European Society of Urogenital Radiology. ESUR prostate MR guidelines 2012. Eur. Radiol. 22, 746-757 (2012).

34. Weinreb JC, Blume JD, Coakley FV, et al. Prostate cancer: sextant localization at MR imaging and MR spectroscopic imaging before prostatectomy-results of ACRIN prospective multi-institutional clinicopathologic study. Radiology. 251, 122-133 (2009).

35. Schulte RT, Wood DP, Daignault S, et al. Utility of extended pattern prostate biopsies for tumor localization: pathologic correlations following radical prostatectomy. Cancer. 113, 1559-1565 (2008). 